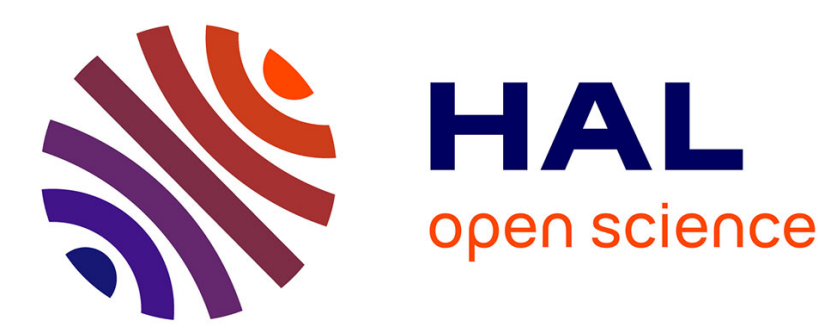

\title{
Combinatorial Characterization of the Language Recognized by Factor and Suffix Oracles
}

Alban Mancheron, Christophe Moan

\section{To cite this version:}

Alban Mancheron, Christophe Moan. Combinatorial Characterization of the Language Recognized by Factor and Suffix Oracles. International Journal of Foundations of Computer Science, 2005, 16 (6), pp.1179-1191. 10.1142/S0129054105003741 . hal-00487228

\section{HAL Id: hal-00487228 \\ https://hal.science/hal-00487228}

Submitted on 28 May 2010

HAL is a multi-disciplinary open access archive for the deposit and dissemination of scientific research documents, whether they are published or not. The documents may come from teaching and research institutions in France or abroad, or from public or private research centers.
L'archive ouverte pluridisciplinaire HAL, est destinée au dépôt et à la diffusion de documents scientifiques de niveau recherche, publiés ou non, émanant des établissements d'enseignement et de recherche français ou étrangers, des laboratoires publics ou privés. 


\title{
COMBINATORIAL CHARACTERIZATION OF THE LANGUAGE RECOGNIZED BY FACTOR AND SUFFIX ORACLES
}

\author{
ALBAN MANCHERON \\ and \\ CHRISTOPHE MOAN \\ Laboratoire d'Informatique de Nantes-Atlantique, \\ Université de Nantes, \\ B.P. 92 208, 44 322 Nantes Cedex 3, France \\ Received (received date) \\ Revised (revised date) \\ Communicated by Editor's name
}

\begin{abstract}
Sequence Analysis requires to elaborate data structures, which allow both an efficient storage and use. A new one was introduced in 1999 by Cyril Allauzen, Maxime Crochemore and Mathieu Raffinot. This structure is linear on the size of the represented word both in time and space. It has the smallest number of states and it accepts at least all substrings of the represented word. This structure is called Factor Oracle. Authors developed another structure on the basis of Factor Oracle, which has the same properties except it accepts at least all suffixes instead of all factors of the represented word. This structure is then called Suffix Oracle. The characterization of the language recognized by the Factor/Suffix Oracle of a given word is an open problem, for which we provide a solution. Using this result, we show that these structures may accept an exponential number of words, which are not factors/suffixes of the given word.
\end{abstract}

Keywords: Factor Oracle, Suffix Oracle, automata, language, characterization.

\section{Introduction}

Several structures have been developed in text indexation: we can cite Tries [1], Suffix Automata [1, 2], Suffix Trees [1,3]... Their objective is to represent a text or a word $s$ (i.e. a succession of symbols taken in an arbitrary alphabet denoted by $\Sigma$ ), in order to "quickly" determine whether this word contains some specific sub-word. This sub-word is then called a factor of $s$.

Allauzen \& al. $[4,5,6]$ described a method allowing to build an acyclic automaton, which accepts at least all factors of $s$, which have as few states as possible $(|s|+1)$ and which is linear in the number of transitions $(2|s|-1)$. When each state is final in this automaton, the structure is called a Factor Oracle. By keeping only "particular" states as final, this automaton becomes a Suffix Oracle. 
Several advantages have been described for this structure. The construction algorithm is easy to understand and implement; such advantage doesn't exist in the most efficient algorithm to build Suffix Trees [3]. Oracles are homogeneous automata, i.e. all transitions ingoing to a same state are labeled with a same symbol. Thus, it is not necessary to label edges anymore. Therefore this structure requires less memory than Suffix Trees or Tries. Lefebvre \& al. [7, 8, 9] used it for repeated motifs discovery over large genomic data and obtained in a few seconds similar results to the ones obtained by using thousands of BLASTn requests. Authors also used the Factor Oracle for text compression [10].

However, at least two open questions are linked to these Oracles: the first one is about the characterization of the language recognized by Oracles; and the second question concerns the existence of a linear algorithm in time and space to build an automaton, which accepts all factors/suffixes of a word $s$ and which is minimal in number of transitions.

When using these Oracles, the main difficulty is to distinguish true and false positives. Therefore we will provide in the next section several definitions related to the construction of Oracles. We will characterize the language recognized by this structure in section 3. Finally, some results using Oracles will also be described.

\section{Definitions}

In the following sections, we call Fact(s) (resp. Suff(s) and Pref(s)) the set of factors (resp. suffixes and prefixes) of $s \in \Sigma^{+}$. We call Pref $f_{s}(i)$ the prefix of $s$, which has length $i \geq 0$. Given $x \in \operatorname{Fact}(s), N b_{s}(x)$ is the number of occurrences of $x$ in $s$ and $x$ is repeated if and only if $N b_{s}(x) \geq 2$.

Definition 1 Given a word $s \in \Sigma^{+}$and $x$ a factor of $s$, we define the function $\underline{\text { Pos }}$ as the position of the first occurrence of $x$ in $s=u x v\left(u, v \in \Sigma^{*}\right): \operatorname{Pos}_{s}(x)=|u|+1$. We also define the function poccur such that $\operatorname{poccur}_{s}(x)=|u x|=\operatorname{Pos}_{s}(x)+|x|-1$.

\subsection{Oracles}

The Oracle construction is defined by the algorithm of Allauzen \& al. [4] (see algorithm 1). Authors gave another algorithm to build the same automaton in linear time on the size of $s$. However, since we are only interested in properties of the Oracle, we do not report it in this paper.

Definition 2 [4] Given a word $s \in \Sigma^{*}$, we define the Factor Oracle of $s$ as the automaton obtained by the algorithm 1, where all states are final. It is denoted by $F O(s)$. We define the Suffix Oracle of $s$ as the automaton obtained by the same algorithm, where a state $e_{i}(0 \leq i \leq|s|)$ is final if and only if there exists a path labeled by a suffix of $s$ from the initial state to the state $e_{i}$. It is denoted by $S O(s)$.

We use the term Oracle to equally designate the Factor or the Suffix Oracle of a word $s$ and we denote it by $O(s)$. We define a relation of order between states in these Oracles. Indeed, if we have two states $e_{i}$ and $e_{j}$ such that $i \leq j$, then $e_{i} \leq e_{j}$.

${ }^{\mathrm{a}}$ As mentioned in [11], the term $-|u|($ line 17) is unfortunately missing in the original algorithm. 
Algorithm 1: Construction of the Factor Oracle of a word ${ }^{a}$

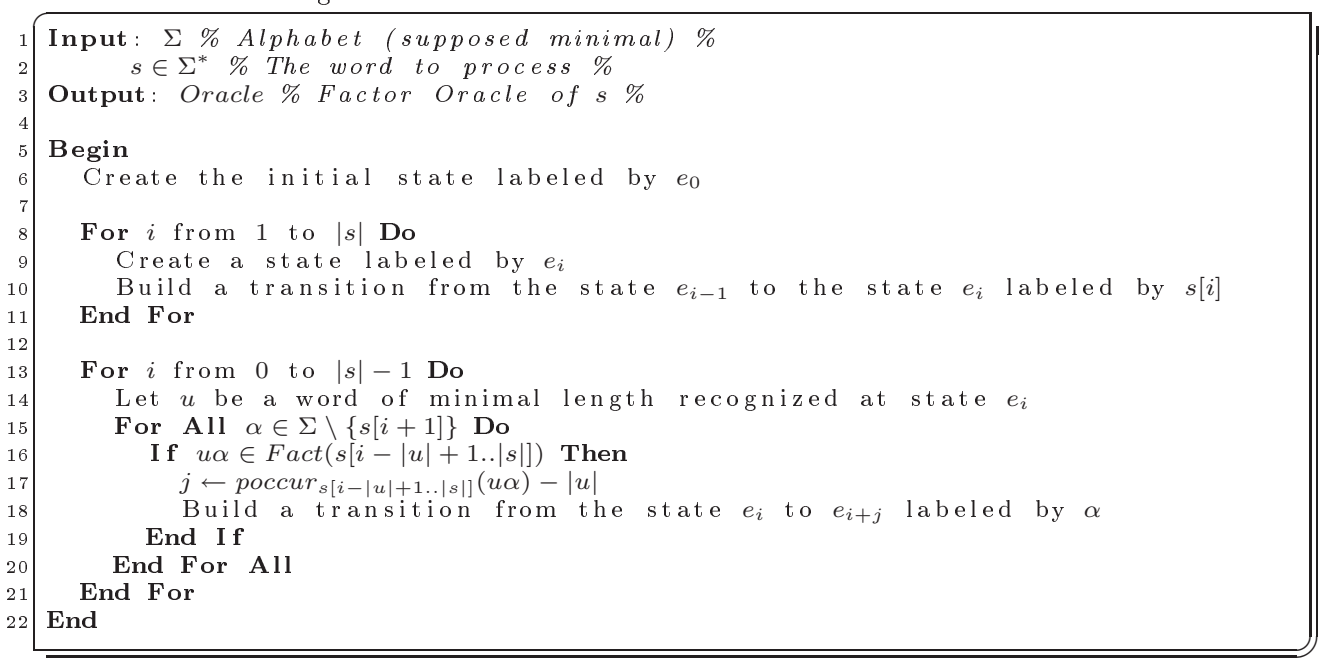

Definition 3 Given a word $s \in \Sigma^{*}$ and a word $x$ accepted at the state $e_{i}(0 \leq i \leq$ $|s|)$ in the Oracle of $s$, we define the function State as State $(x)=e_{i}$.

Lemma 1 [4] Given a word $s \in \Sigma^{*}$, a unique word with minimal length is accepted at each state $e_{i}(0 \leq i \leq|s|)$ in the Oracle of $s$. It is denoted by $\min \left(e_{i}\right)$.

Lemma 2 [4] Given a word $s \in \Sigma^{*}$, its Oracle and an integer $i(0 \leq i \leq|s|)$, then $\min \left(e_{i}\right) \in \operatorname{Fact}(s)$ and $i=\operatorname{poccur}_{s}\left(\min \left(e_{i}\right)\right)$.

Notation 1 Given a word $s \in \Sigma^{*}$, let $\#_{i n}\left(e_{i}\right)$ and $\#_{\text {out }}\left(e_{i}\right)$ denote the number of ingoing/outgoing transitions to/from state $e_{i}(0 \leq i \leq|s|)$ in the Oracle of $s$.

\subsection{Canonical Factors \& Contraction Operation}

In this section, we will define particular factors from a given word and an operation needed to characterize the language. Then we will define the sets of words obtained with this operation.

Definition 4 Given a word $s \in \Sigma^{*}$ and its Oracle, we define the set of Canonical Factors of $s$ as $\mathcal{F}_{s}=\left\{\min \left(e_{i}\right)|1 \leq i \leq| s \mid \wedge\left(\#_{\text {out }}\left(e_{i}\right)>1 \vee \#_{\text {in }}\left(e_{i}\right)>\overline{1)\}}\right.\right.$. Given a suffix $t$ of $s$ and a Canonical Factor $f$ of $s, f$ is a conserved Canonical Factor of $s$ in $t$ if the first occurrence of $f$ in $s$ appears in $t$. The set of the conserved Canonical Factors of $s$ in $t$ is denoted by $\mathcal{F}_{s, t}\left(\right.$ thus $\left.\mathcal{F}_{s, t} \subseteq \mathcal{F}_{s}\right)$.

Definition 5 Given a word $s \in \Sigma^{*}$ and a repeated Canonical Factor $f$ of $s$ such that:

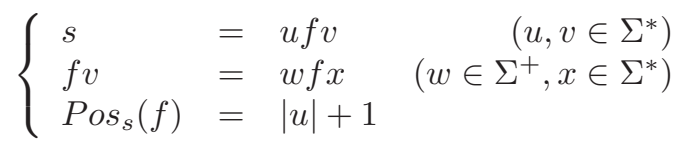

then the pair $(|u|+1,|u w|+1)$ is a contraction of $s$ by $f$. 
Notation 2 Given a word $s \in \Sigma^{*}$ and a Canonical Factor $f \in \mathcal{F}_{s}, \mathcal{C}_{s}^{f}$ is the set of contractions of $s$ by $f$ and $\mathcal{C}_{s}^{*}\left(\equiv \bigcup_{f \in \mathcal{F}_{s}} \mathcal{C}_{s}^{f}\right)$ is the set of all the contractions we can apply to $s$. Given a suffix $t$ of $s=t^{\prime} t\left(t, t^{\prime} \in \Sigma^{*}\right)$, then $\mathcal{C}_{s, t}^{*}$ is the subset of $\mathcal{C}_{s}^{*}$ such that $\mathcal{C}_{s, t}^{*}=\left\{(p, q)\left|(p, q) \in \mathcal{C}_{s}^{*} \wedge p>\right| t^{\prime} \mid\right\}$.

In the following, we will use sets of contractions to produce new words from a given one. Then we will use these words to characterize the language recognized by Oracles.

Definition 6 A set $\mathcal{C}$ of contractions is coherent if and only if it does not contain two contractions $\left(i_{1}, j_{1}\right),\left(i_{2}, j_{2}\right)$ such that: $i_{1}<i_{2}<j_{1}<j_{2}$. Furthermore, $\mathcal{C}$ is $\underline{\text { minimal }}$ if and only if it does not contain two contractions $\left(i_{1}, j_{1}\right)$ and $\left(i_{2}, j_{2}\right)$ such that $i_{1} \leq i_{2}<j_{2} \leq j_{1}$ or such that $i_{1}<j_{1}=i_{2}<j_{2}$.

Definition 7 Given a word $s \in \Sigma^{*}$ and a coherent and minimal set of contractions $\mathcal{C}=\left\{\left(p_{1}, q_{1}\right), \ldots,\left(p_{k}, q_{k}\right)\right\}$ (associated to the set of canonical factors $\left\{f_{1}, \ldots, f_{k}\right\}$ ), then we define the function Word as following:

$W \operatorname{ord}(s, \mathcal{C})=s\left[1 . . p_{1}-1\right] s\left[q_{1} . . p_{2}-1\right] \ldots s\left[q_{k-1} . . p_{k}-1\right] s\left[q_{k} . .|s|\right]$

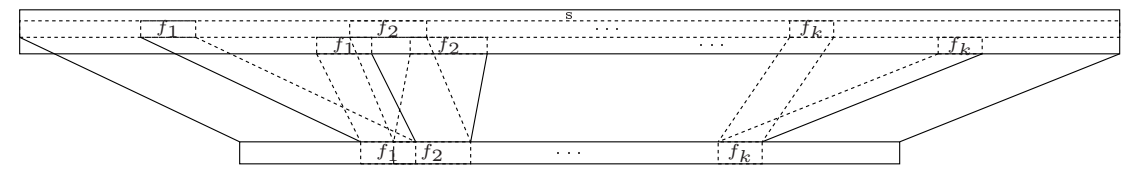

Fig. 1. Words obtained using the contraction operation (see Definition 5).

We are only interested by words obtained by the contraction operation. So we will only consider coherent and minimal sets of contractions without loss of generality. Note that the word remains the same whatever the order of contractions (see figure 1).

Definition 8 We define $\mathcal{E}(s)=\bigcup_{\mathcal{C} \subseteq \mathcal{C}_{s}^{*}} W \operatorname{ord}(s, \mathcal{C})$, as the closure of $s$.

Example: Consider the word gaccattctc (see figure 2). Its set of Canonical Factors is $\mathcal{F}_{\text {gaccattctc }}=\{a, c, c a, t, t c, c t\}$ and then $\mathcal{C}_{\text {gaccattctc }}^{*}=\{(2,5),(3,4),(3,8),(3,10)$, $(6,7),(6,9),(7,9)\}$. Now, consider the set $\mathcal{C}=\{(2,5),(7,9)\}\left(\mathcal{C} \subseteq \mathcal{C}_{\text {gaccattctc }}^{*}\right)$, then Word $($ gaccattctc, $\mathcal{C})=$ gackattctc $=$ gattc. The closure of gaccattctc is:

$\mathcal{E}($ gaccattctc $)=\left\{\begin{array}{l}\text { gac, gacatc, gacatctc, gacattc, gacattctc, gaccatc, } \text { gaccatctc }, \\ \text { gaccattc, } \text { gaccattctc, gactc, gatc, gatctc, gattc, gattctc }\end{array}\right\}$.

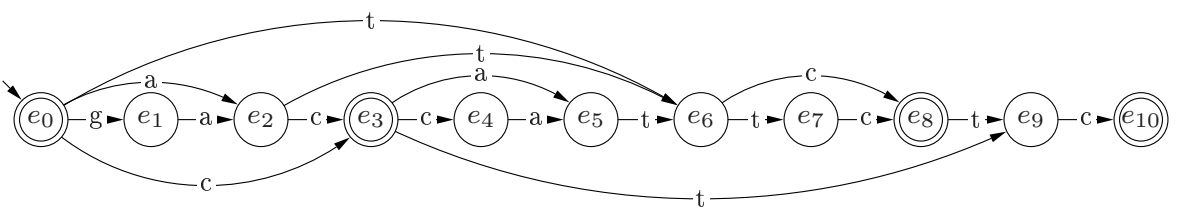

Fig. 2. Suffix Oracle of gaccattctc. In the Factor Oracle, all states are final. 


\section{Characterization of the language recognized by Oracles}

Given a word $s \in \Sigma^{*}$, we saw how to build the corresponding Factor (resp. Suffix) Oracle. This Oracle allows to recognize the factors (resp. suffixes) of $s$, but it also accepts additional words. For example the word atc is accepted by the Factor (resp. Suffix) Oracle of gaccattctc (see figure 2), whereas it is neither a factor nor a suffix of this sequence. We will see that the Suffix Oracle of $s$ exactly recognizes all suffixes of words from $\mathcal{E}(s)$ and we will use this result to prove that the Factor Oracle of $s$ exactly recognizes all factors of words from $\mathcal{E}(s)$.

We use following Lemmas to prove the result concerning Suffix Oracles. These Lemmas have been proved in [4].

Lemma 3 [4] Given a word $s \in \Sigma^{*}$ and an integer $i(0 \leq i \leq|s|)$, then $\min \left(e_{i}\right)$ is suffix of all words recognized at state $e_{i}$ in the Oracle of $s$.

Lemma 4 [4] Given a word $s \in \Sigma^{*}$ and a factor $w$ of $s$, then $w$ is recognized at state $e_{i}\left(1 \leq i \leq \operatorname{poccur}_{s}(w)\right)$ in the Oracle of $s$.

Lemma 5 [4] Given a word $s \in \Sigma^{*}$ and an integer $i(0 \leq i \leq|s|)$, then every path ending by $\min \left(e_{i}\right)$ in the Oracle of $s$ leads to a state $e_{j}$ such that $j \geq i$.

Lemma 6 [4] Given a word $s \in \Sigma^{*}$ and $w \in \Sigma^{*}$ a word accepted at state $e_{i}(0 \leq$ $i \leq|s|)$ in the Oracle of $s$, then every suffix of $w$ is also recognized by the Oracle at state $e_{j}$ such that $j \leq i$.

The proof of Lemma 6 is only given in [4] for Factor Oracles. We need this result to be true for Suffix Oracles.

Proof. The original Lemma gives us that if $x$ is a suffix of $w$, then State $(x) \leq$ $\operatorname{State}(w)$. We need to prove that if State $(w)$ is final, then State $(x)$ is final. Therefore, we have to consider two cases. When $|x| \geq\left|\min \left(e_{i}\right)\right|$, we have $\min \left(e_{i}\right) \in$ $\operatorname{Suff}(x)$ and thus, according to Lemma $5, \operatorname{State}(x) \geq \operatorname{State}\left(\min \left(e_{i}\right)\right)$. Since $\operatorname{State}\left(\min \left(e_{i}\right)\right)=e_{i}=\operatorname{State}(w)$, we conclude that $\operatorname{State}(x)=\operatorname{State}(w)$. When we have $|x|<\left|\min \left(e_{i}\right)\right|$, since the state $e_{i}$ is final, then there exists a suffix $t$ of $s$ such that State $(t)=e_{i}$. According to Lemma 3, we conclude that $\min \left(e_{i}\right) \in$ $\operatorname{Suff}(t) \subseteq \operatorname{Suff}(s)$. Since $x$ and $\min \left(e_{i}\right)$ are suffixes of $w$, then $|x|<\left|\min \left(e_{i}\right)\right| \Rightarrow$ $x \in \operatorname{Suff}\left(\min \left(e_{i}\right)\right)$. So $x$ is also suffix of $s$ and according to Definition of the Suffix Oracle $\operatorname{State}(x)$ is final.

We use these previous results to show that the two following Lemmas.

Lemma 7 Given a word $s \in \Sigma^{*}$, a Canonical Factor $f$ of $s=u f v\left(u, v \in \Sigma^{*}\right)$ such that $f$ is not repeated in uf and a set $\mathcal{C}$ of contractions $\left(\mathcal{C} \in \mathcal{C}_{s}^{*}\right)$ such that $W o r d(u f, \mathcal{C})=w f$, then the Oracle of s accepts $w f$ and $f$ at the same state.

Proof. We denote by $\mathcal{C}_{i} \subseteq \mathcal{C}_{s}^{*}$ a set of contractions, which has cardinality $i$. In the same way, we denote by $w_{i} f$ the word obtained when we apply contractions $\mathcal{C}_{i}$ to uf (warning: $w_{i} f=\operatorname{Word}\left(u f, \mathcal{C}_{i}\right) \nRightarrow w_{i}=\operatorname{Word}\left(u, \mathcal{C}_{i}\right)$ ). By induction on the size of $\mathcal{C}_{i}$, we show that $\operatorname{State}\left(\operatorname{Word}\left(u f, \mathcal{C}_{i}\right)\right)=\operatorname{State}(f)$ for all $\mathcal{C}_{i} \in \mathcal{C}_{s}^{*}$.

Let $e_{x}=\operatorname{State}(f)\left(f=\min \left(e_{x}\right)\right.$ by Definition of $\left.f\right)$ and $e_{x_{i}^{\prime}}=\operatorname{State}\left(\operatorname{Word}\left(u f, \mathcal{C}_{i}\right)\right)$. If we consider $\mathcal{C}_{0}$, then $\operatorname{Word}\left(u f, \mathcal{C}_{0}\right)=u f$. According to Lemma $5, x_{0}^{\prime} \geq x$. Furthermore, according to Lemma 4 applied to $u f$, we have $x_{0}^{\prime} \leq \operatorname{poccur}_{s}(u f)$. 
However by Definition of $f$, $\operatorname{poccur}_{s}(f)=|u f|=\operatorname{poccur}_{s}(u f)$. Therefore we have $x_{0}^{\prime} \leq x$ and $x_{0}^{\prime}=x$.

If this lemma is true for a set $\mathcal{C}_{i} \subset \mathcal{C}_{s}^{*}$ of contractions, then it is true for a set $\mathcal{C}_{i+1}=$ $\mathcal{C}_{i} \cup\{(p, q)\}$. We assume without loss of generality (see figure 1 ) that $(p, q)$ is the last contraction in $\mathcal{C}_{i+1}$ (by ascending order over positions). Let $b$ be the Canonical Factor used by this contraction. We can write $u f=s[1 . . p-1] s[p . . q-1] s[q . .|u f|]$. Since $(p, q)$ is chosen as the last contraction, all contractions in $\mathcal{C}_{i}$ are applicable to $s[1 . . p-1]$. So we define $a, c \in \Sigma^{*}$ such that $w_{i} f=a s[p . .|u f|]=a b c$ and $d \in \Sigma^{*}$ such that $w_{i+1} f=a s[q . .|u f|]=a b d$. Also $a b=\operatorname{Word}\left(s[1 . . p-1] b, \mathcal{C}_{i}\right)$ : the opposite would mean that contraction $(p, q)$ can't be operated from $b$ and according to the induction hypothesis, State $(a b)=\operatorname{State}(b)$. From this, we conclude that $\operatorname{State}(a b c)=\operatorname{State}(b c)$ and State $(a b d)=\operatorname{State}(b d)$. We know that $b d(=s[q . .|u f|])$ is a suffix of $b c(=s[p . .|u f|])$ and according to Lemma 6 :

$$
\begin{aligned}
& \text { State }(b d) \leq \text { State }(b c) \\
& \Leftrightarrow \operatorname{State}(a b d) \leq \text { State }(a b c) \\
& \Leftrightarrow \operatorname{State}\left(w_{i+1} f\right) \leq \operatorname{State}\left(w_{i} f\right) \\
& \Leftrightarrow \operatorname{State}\left(w_{i+1} f\right) \leq \operatorname{State}(f)
\end{aligned}
$$

However, according to Lemma 5 , we have State $\left(w_{i+1} f\right) \geq \operatorname{State}(f)$ and therefore $\operatorname{State}\left(w_{i+1} f\right)=\operatorname{State}(f)$. This lemma is then true for all $\mathcal{C}_{i} \subseteq \mathcal{C}_{s}^{*}$.

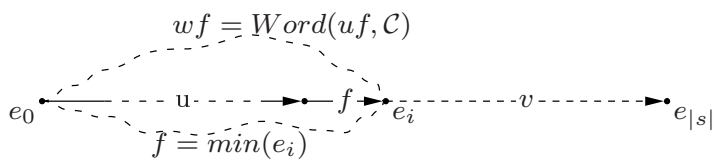

Fig. 3. Illustration of Lemma 7.

Now, we show how to obtain a contraction, starting with transition of type $e_{i} \rightarrow e_{j}$ with $j>i+1$ (that we call an external transition in the subsequent).

Lemma 8 Given a word $s \in \Sigma^{*}$ and an integer $i(0 \leq i<|s|)$ such that $\#_{\text {out }}\left(e_{i}\right)>$ 1 , let $p$ be a external transition from state $e_{i}$ to state $e_{i+j}(j>1)$ labeled by $\alpha$ and $u=\min \left(e_{i}\right)$. Then an occurrence of $u \alpha$ exists at position $(i+j-|u|)$ of $s$ (see figure 5 - page 8). Moreover, the contraction $(i-|u|+1, i-|u|+j)$ of $s$ by $u$ exists too.

Proof. According to the construction algorithm (see algorithm 1), the transition $p$ is added from $e_{i}$ to $e_{i+j}$ because a position $j$ in $s[i-|u|+1 . .|s|]$ is such that: $j=$ $\operatorname{poccur}_{s[i-|u|+1 . .|s|]}(u \alpha)-|u|$. We also have $u \alpha \in$ Fact(s) because $u \alpha \in$ Fact $(s[i-$ $|u|+1 . .|s|])$. CLeOphas \& al. [11] proved that since $u=\min \left(e_{i}\right)$ and $u \alpha \in \operatorname{Fact}(s)$, then $i-|u|+\operatorname{poccur}_{s[i-|u|+1 . .|s|]}(u \alpha)=\operatorname{poccur}_{s}(u \alpha)$. We have $i+j=\operatorname{poccur}_{s}(u \alpha)$ and finally $s[i+j-|u|, i+j]=u \alpha$.

We use algorithm Contractor (see algorithm 2) to give a characterization of the language accepted by the Oracle of a word $s$. Given a word $s \in \Sigma^{*}$ and its Suffix Oracle $S O(s)$, initial inputs of Contractor are a word $w$ accepted by $S O(s)$ and $t$, the maximal suffix of $s$ beginning with $w[1]$. This algorithm outputs a set $\mathcal{C} \in \mathcal{C}_{s}^{*}$ of contractions such that $w=W \operatorname{ord}(t, \mathcal{C})$. 
Algorithm 2: Contractions needed to transform $s=t^{\prime} t\left(t, t^{\prime} \in \Sigma^{*}\right)$ into $t^{\prime} w$

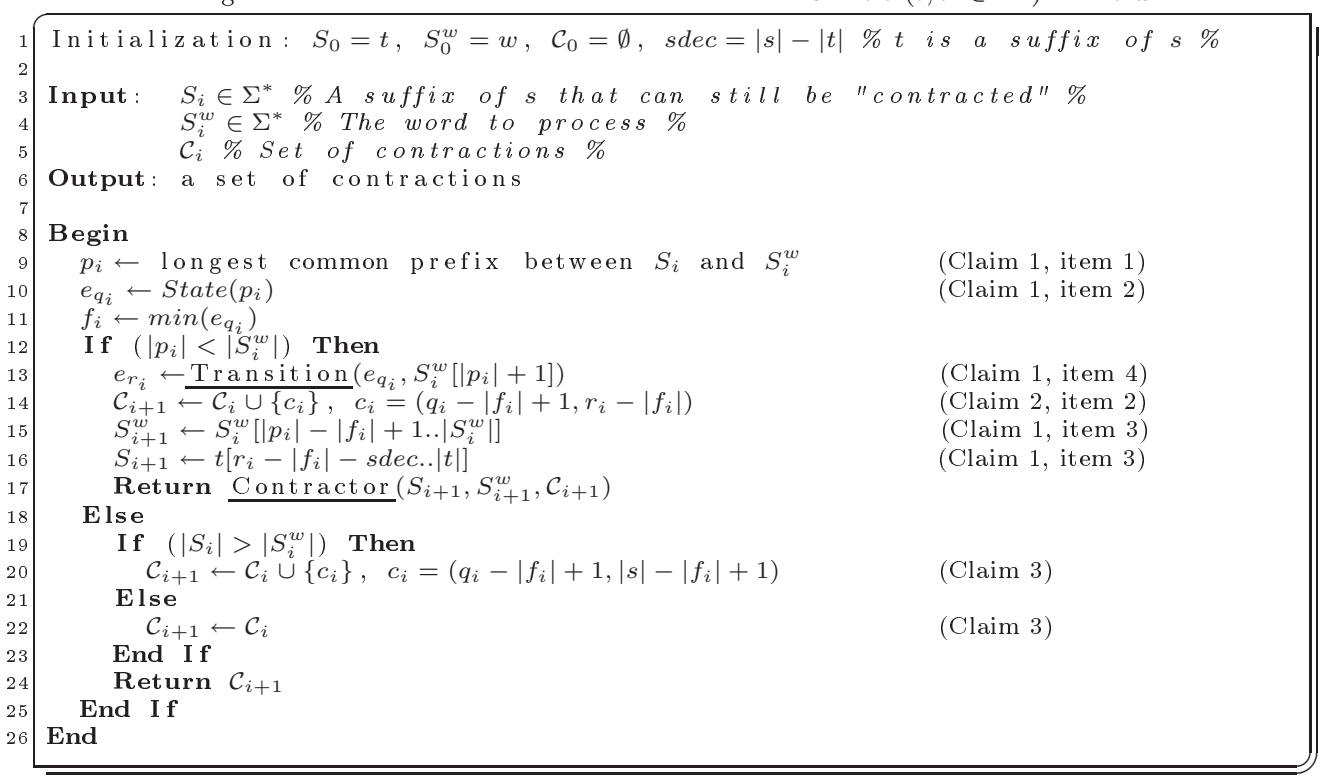

By Definition 7 , given a word $s \in \Sigma^{*}$ such that $s=t^{\prime} t\left(t, t^{\prime} \in \Sigma^{*}\right)$ and a set $C \in \mathcal{C}_{s, t}^{*}$ of contractions, $t^{\prime} w=W \operatorname{ord}(s, C)$ is then a concatenation of substrings of $s$. These substrings can be assimilated as prefixes of suffixes of $s$. A contraction is then a "jump" from one substring to the next one. The main idea of this algorithm is to read from left to right the sequences $t$ and $w$, in order to compute the longest common prefixes between given suffixes of $t$ and $w$. After each stage, the algorithm computes the length of the "jump" to go to the next suffix of $t$ to consider.

Inputs are words $S_{i}, S_{i}^{w}(i \geq 0)$ and a set $\mathcal{C}_{i}$ of contractions. We initialize $S_{0}=t$, $S_{0}^{w}=w, \mathcal{C}_{0}=\emptyset$ and $p_{i}$ (line 9 ) as the longest prefix of $S_{i}$ and $S_{i}^{w}$. So:

$$
S_{i}=p_{i} S_{i}^{\prime} \quad \text { and } \quad S_{i}^{w}=p_{i} S_{i}^{w} .
$$

Let $e_{q_{i}}=\operatorname{State}\left(p_{i}\right)$ and $f_{i}=\min \left(e_{q_{i}}\right)$ ((lines 10 and 11), Lemma 3 provides:

$$
p_{i}=p_{i}^{\prime} f_{i} \quad\left(p_{i}^{\prime} \in \Sigma^{*}\right) .
$$

The variable $e_{r_{i}}$ (line 13) is the state reached by the transition from $e_{q_{i}}$ and labeled by $\alpha=S_{i}^{w}\left[\left|p_{i}\right|+1\right]=S_{i}^{\prime w}[1]$. The set $\mathcal{C}_{i+1}$ has cardinality $i+1$. The variable $s d e c=|s|-|t|$ is necessary to compute $S_{i+1}$ (line 15), because each state $e_{i}$ is linked to the $i^{t h}$ character of $s$, not to the character $(i-|s|+|t|)$ of $t$.

Claim 1 Following assertions are true for all $i \geq 0$ (see figure 4 ):

1. $f_{i} \alpha \in \operatorname{Pref}\left(p_{i+1}\right)$.

2. $S_{i}=t\left[q_{i}-\left|p_{i}\right|+1-\right.$ sdec.. $\left.|t|\right]$.

3. $S_{i+1}$ and $S_{i+1}^{w}$ are respectively suffixes of $S_{i}$ and $S_{i}^{w} ; S_{i}$ and $S_{i}^{w}(i \geq 0)$ are respectively suffixes of $t$ and $w$.

4. Transition from $e_{q_{i}}$ to $e_{r_{i}}$ labeled by $\alpha$ always exists. 

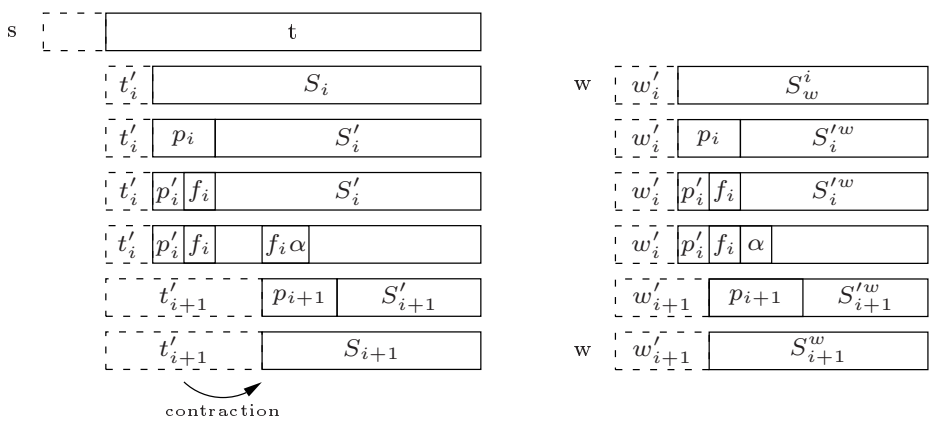

Fig. 4. Visualization of Contractor on $S_{i}$ and $S_{i}^{w}$.

\section{Proof.}

1. Since $f_{i}=\min \left(e_{q_{i}}\right)$ and according to Lemma 8 , we have $s\left[r_{i}-\left|f_{i}\right| . . r_{i}\right]=$ $t\left[r_{i}-\left|f_{i}\right|-\right.$ sdec.. $\left.r_{i}-s d e c\right]=f_{i} \alpha$. So $S_{i+1}$ and $S_{i+1}^{w}$ begin with $f_{i} \alpha$ (line 15).

2. For $i=0$ (initialization case), $S_{0}=t$ and $t$ is the longest suffix of $s$ beginning by $w[1]$. If $e_{x}=\operatorname{State}\left(S_{0}[1]\right)(x>0)$, then $t[x-\operatorname{sdec} .|t|]=S_{0}$ and $\operatorname{State}\left(p_{0}\right)=x+\left|p_{0}\right|-1=e_{q_{i}}$. Thus we conclude that $S_{0}=s\left[q_{0}-\left|p_{0}\right|+1-\right.$ sdec.. $\left.|s|\right]$. At iteration $i$, we have $S_{i+1}=t\left[r_{i}-\left|f_{i}\right|-\right.$ sdec..|t|] (line 16). Since $S_{i+1}^{w}$ begins by $f_{i} \alpha$ (item 1 ), we conclude that $q_{i+1}=r_{i}+\left|p_{i+1}\right|-\left|f_{i}\right|-1$ and finally we have $S_{i+1}=t\left[r_{i}-\left|f_{i}\right|-s d e c . .|t|\right]=t\left[q_{i+1}-\left|p_{i+1}\right|+1-\right.$ sdec.. $\left.|t|\right]$.

3. This assertion is true for $S_{i}^{w}$ because $S_{i+1}^{w}$ is suffix of $S_{i}^{w}$ by construction (line 15) and $S_{0}^{w}=w$. Concerning $S_{i}$, we have $S_{0}=t$ thus the property is true for $i=0$. Suppose that $S_{i}$ is suffix of $t$, from item 2 , we have $S_{i}=t\left[q_{i}-\left|p_{i}\right|+1-\right.$ sdec.. $\left.|t|\right]$. We also have $S_{i+1}=t\left[r_{i}-\left|f_{i}\right|-s d e c . .|t|\right]$ (line 16). So we conclude using Eq. (2) that $q_{i}-\left|p_{i}\right|=q_{i}-\left|p_{i}^{\prime}\right|-\left|f_{i}\right|$. Since $\left|p_{i}^{\prime}\right| \geq 0$, we have $q_{i}-\left|f_{i}\right| \geq q_{i}-\left|p_{i}\right|$ and $r_{i}>q_{i}$. Finally, we conclude that $r_{i}-\left|f_{i}\right|>q_{i}-\left|p_{i}\right|$ and that $S_{i+1}$ is a suffix of $S_{i}$.

4. According to item $3, S_{i}^{w}$ is suffix of $w$. Then $S_{i}^{w}$ is accepted by $O(s)$ (Lemma 6). Using Eq. (1) with $S_{i}^{\prime w}[1]=\alpha$ the transition must exist and implies $\#_{\text {out }}\left(e_{q_{i}}\right) \geq 2$. So $f_{i}=\min \left(e_{q_{i}}\right) \in \mathcal{F}_{s}$ by Definition of Canonical Factors.

From Eq. (1) and Claim 1 (item 4):

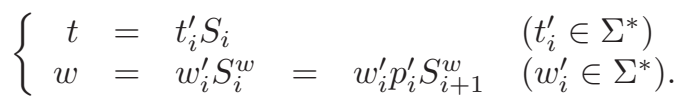

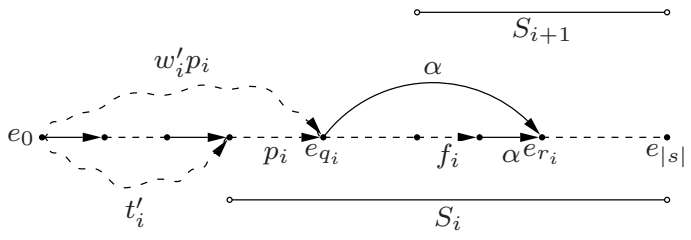

Fig. 5. Illustration of a step in the algorithm $\underline{\text { Contractor }}\left(\alpha=S_{i}^{w}\left[\left|p_{i}\right|+1\right]\right)$.

Claim 2 For all $i \geq 0$ (see figure 5):

1. $\operatorname{State}\left(w_{i}^{\prime} p_{i}\right)=\operatorname{State}\left(t_{i}^{\prime} p_{i}\right)=\operatorname{State}\left(p_{i}\right)=e_{q_{i}}$.

2. $c_{i}$ is a contraction of $t_{i}^{\prime} S_{i}$ (resp. $w_{i}^{\prime} S_{i}$ ) provided by $f_{i}$. Result of this contraction is $t_{i}^{\prime} p_{i}^{\prime} S_{i+1}$ (resp. $\left.w_{i}^{\prime} p_{i}^{\prime} S_{i+1}=w_{i+1}^{\prime} S_{i+1}\right)$. 


\section{Proof.}

1. For $i=0, t_{i}^{\prime}=w_{i}^{\prime}=\epsilon$. Let us suppose that the property is true for $i$ and therefore for $i+1$. From Claim 1 (item 2), we conclude that $S_{i}$ labeled a path using only "main" transitions (i.e. transitions of type $e_{j} \rightarrow e_{j+1}$ ) from $e_{q_{i}-\left|p_{i}\right|}$ to $e_{|s|}$ in $O(s)$. According to Claim 1 (item 3) we conclude that:

$$
S_{i}=u S_{i+1} \quad\left(u \in \Sigma^{*}\right) .
$$

So, the state $e_{x}\left(x>q_{i}-\left|p_{i}\right|\right)$ is such that $S_{i+1}$ labeled a path using only "main" transitions from $e_{x}$ to $e_{|s|}$ in $O(s)$ and more precisely $x=r_{i}-\left|f_{i}\right|-1$. We have $t_{i+1}^{\prime}=t_{i}^{\prime} u$ (Eq. (3) and (4)) and State $\left(t_{i}^{\prime} u\right)=e_{x}$. Since $f_{i}=\min \left(e_{q_{i}}\right)$ and there is a transition from $e_{q_{i}}$ to $e_{r_{i}}$ labeled by $\alpha$ (Claim 1, item 4$)$, State $\left(t_{i+1}^{\prime} f_{i} \alpha\right)=$ $\operatorname{State}\left(t_{i}^{\prime} u f_{i} \alpha\right)=\operatorname{State}\left(f_{i} \alpha\right)=e_{r_{i}}$ and furthermore, $p_{i+1}=f_{i} \alpha v\left(v \in \Sigma^{*}\right)$. So we have State $\left(t_{i+1}^{\prime} f_{i} \alpha v\right)=\operatorname{State}\left(t_{i+1}^{\prime} p_{i+1}\right)=\operatorname{State}\left(p_{i+1}\right)$.

2. From Eq. (1), (2) and (3), we conclude that:

$$
t=t_{i}^{\prime} S_{i}=t_{i}^{\prime} p_{i}^{\prime} f_{i} S_{i}^{\prime}
$$

Since $S_{i+1} \in \operatorname{Suff}\left(S_{i}\right)$, we have $S_{i}=u S_{i+1}\left(u \in \Sigma^{+}\right)$and from Eq. (5), $t_{i}^{\prime} p_{i}^{\prime} f_{i} S_{i}^{\prime}=$ $t_{i}^{\prime} u S_{i+1}$. According to Claim 1 (item 1), we have $t_{i}^{\prime} p_{i}^{\prime} f_{i} S_{i}^{\prime}=t_{i}^{\prime} u f_{i} \alpha u^{\prime}\left(u^{\prime} \in \Sigma^{*}\right)$. Since we have $\operatorname{State}\left(t_{i}^{\prime} p_{i}^{\prime} f_{i}\right)=\operatorname{State}\left(f_{i}\right)$ and $|u|>\left|p_{i}^{\prime}\right|$ (because $S_{i}^{\prime}[1] \neq \alpha$ ), we can contract $t_{i}^{\prime} S_{i}$ by $f_{i}$ and $t_{i}^{\prime} p_{i}^{\prime} f_{i} \alpha u^{\prime}=t_{i}^{\prime} p_{i}^{\prime} S_{i+1}$. We can conclude that $w_{i}^{\prime} S_{i}=w_{i}^{\prime} p_{i} S_{i}^{\prime}$ is contracted by $f_{i}$ in $w_{i}^{\prime} p_{i}^{\prime} S_{i+1}$ because State $\left(w_{i}^{\prime} p_{i}\right)=\operatorname{State}\left(t_{i}^{\prime} p_{i}\right)$. According to Eq. (3), we conclude that $w_{i+1}^{\prime}=w_{i}^{\prime} p_{i}^{\prime}$ and $w_{i}^{\prime} p_{i}^{\prime} S_{i+1}=w_{i+1}^{\prime} S_{i+1}$.

Claim 2 shows that a contraction $c_{i}$ of $t_{i}^{\prime} S_{i}$ by $f_{i}$ is also a contraction for $t$ and for $w_{i}^{\prime} S_{i}$. Consider the $i^{\text {th }}$ iteration, we have $\left|S_{i}^{w}\right|>\left|S_{i+1}^{w}\right|$ or $\left|S_{i}^{w}\right|=\left|S_{i+1}^{w}\right|$ and $\left|p_{i+1}\right|>\left|p_{i}\right|$ (if $f_{i}=p_{i}$ ). Since $p_{i}>0$, we ensure that recursion stops at iteration $j>i$ with $p_{j}=S_{j}^{w}(j>i)$.

Claim 3 Given an integer $i \geq 0$ such that $p_{i}=S_{i}^{w}$, then $t$ needs a last contraction if and only if $\left|S_{i}^{w}\right| \neq\left|S_{i}\right|$.

Proof. The word obtained with the set $\mathcal{C}_{i}$ of contractions is $w_{i}^{\prime} p_{i} S_{i}^{\prime}$ (Claim 2, item 2). If $S_{i}^{w}=S_{i}$, then we have $S_{i}^{\prime}=\epsilon$ and we conclude that $\mathcal{C}_{i}$ is complete (line 20). If $S_{i}^{w} \neq S_{i}$, according to Claim 2 (item 1), we have $\operatorname{State}\left(w_{i}^{\prime} p_{i}\right)=e_{q_{i}}$. By Definition of the final state in a Suffix Oracle, $\min \left(e_{q_{i}}\right) \in \operatorname{Suff}(w)$ (Lemma 3) and $\min \left(e_{q_{i}}\right) \in \operatorname{Suff}(t)$. A last contraction is needed to complete the set of contractions (line 22).

Considering the $i^{\text {th }}$ call of Contractor, inputs are $S_{i}=p_{i} S_{i}^{\prime}, S_{i}^{w}=p_{i} S_{i}^{\prime w}$ and $\mathcal{C}_{i}$. This set is the set of contractions necessary to transform $t_{i}^{\prime} \in \operatorname{Pref}(t)$ into $w_{i}^{\prime} \in \operatorname{Pre} f(w)$. The variable $p_{i}$ refers to the longest common prefix of $S_{i}$ and $S_{i}^{w}$ ( $p_{i}$ is both a factor of $t$ and $w$, Claim 1 ). Two cases may occur for this call (line 12 . When $\left|p_{i}\right|=\left|S_{i}^{w}\right|$, the recursion ends. Otherwise, $\left|p_{i}\right| \neq\left|S_{i}^{w}\right|$ and at least another contraction is necessary until $\left|p_{j}\right|=\left|S_{j}^{w}\right|(j>i)$. From Claim 2, the $i^{\text {th }}$ contraction allows to continue with the suffix $S_{i+1}$. At the end of the process (i.e. the end of $w)$, we return the set $\mathcal{C}=\mathcal{C}_{j}$ and Lemma 9 ensures that $w=\operatorname{Word}(t, \mathcal{C})$. 
Lemma 9 Given a word $s \in \Sigma^{*}$, its Suffix Oracle, a word $w \in \Sigma^{*}$ accepted by $S O(s)$ and the longest suffix $t$ of $s$ such that $w[1]=t[1]$, then Contractor $(t, w, \emptyset)$ outputs a set $\mathcal{C}$, which is such that $w=W \operatorname{ord}(t, C)$.

Proof. Let $i \geq 0$ such that $S_{i+1}^{w}=p_{i+1}$. According to Claim 2, we conclude that $\mathcal{C}_{i+1}$ is a coherent set of contractions of $t$. Since $p_{i+1}$ is prefix of $S_{i+1}$ we have:

$$
W \operatorname{ord}\left(t, \mathcal{C}_{i+1}\right)=w_{i+1}^{\prime} S_{i+1}=w_{i+1}^{\prime} S_{i+1}^{w} u=w_{i+1}^{\prime} p_{i+1} u \quad\left(u \in \Sigma^{*}\right)
$$

If $u=\epsilon$, we have $W \operatorname{ord}\left(t, \mathcal{C}_{i+1}\right)=w($ Eq. (3)). Else (Claim 3) a ultimate contraction $c_{i+1}$ by $f_{i+1}$ transforms $w_{i+1}^{\prime} S_{i+1}^{w} u$ into $w_{i+1}^{\prime} S_{i+1}^{w}=w=\operatorname{Word}\left(t, \mathcal{C}_{i+1} \cup\left\{c_{i+1}\right\}\right)$. Finally Contractor provides a set $\mathcal{C}$ such that $w=W \operatorname{ord}(t, \mathcal{C})$.

We can notice that:

1. $\mathcal{C}$ is not always minimal.

2. $\mathcal{C}$ is coherent. Let $(a, b)$ and $(c, d)$ be two contractions successively added to $\mathcal{C}$. We have $a<b$ and $c<d$ because $r_{i}>q_{i}$ and $|s|>q_{i}$ (lines 14 and 20). If $e_{q_{i+1}}=\operatorname{State}\left(p_{i+1}\right)=e_{r_{i}}$, then $b=c$, else $\left.p_{i+1}=f_{i} \alpha v\left(\alpha=S_{[}^{w}\left|p_{i}\right|+1\right], v \neq \epsilon\right)$ and $e_{q_{i+1}}>e_{r_{i}}$, therefore $b<c$.

Following theorems are the main purpose of this paper:

Theorem 1 Exactly all suffixes of words from $\mathcal{E}(s)$ are recognized by the Suffix Oracle of $s$.

Proof.

' $\Rightarrow$ ': Each suffix of words from $\mathcal{E}(s)$ is recognized by the Suffix Oracle of $s$.

According to Lemma 6 , if $w$ is accepted by $S O(s)$, each suffix of $w$ is also accepted by $S O(s)$. So we only need to prove that each word from $\mathcal{E}(s)$ is accepted by $S O(s)$. Let $\mathcal{C} \in \mathcal{C}_{s}^{*}$ be a set of contractions applicable to $s$ and $w=\operatorname{Word}(s, \mathcal{C})$. The set $\mathcal{C}_{i}$ is the set of the first $i$ contractions of $\mathcal{C}$ (chosen without loss of generality by ascending order over positions - see figure 1$),\left(x_{j}, y_{j}\right)$ is the $j^{\text {th }}$ contraction, which use the Canonical Factor $f_{j}(1 \leq j \leq i)$ and $w_{j}=\operatorname{Word}\left(s, \mathcal{C}_{j}\right)$. The property $(P)$ to prove is that if $w_{i}(0 \leq i<|\mathcal{C}|)$ is accepted by $S O(s)$, then $w_{i+1}$ is accepted too. We have:

$$
\begin{cases}w_{i} & =s\left[1 . . x_{1}-1\right] s\left[y_{1} . . x_{2}-1\right] \ldots s\left[y_{i} . .|s|\right] \\ s\left[y_{i} . . y_{i}+\left|f_{i}\right|-1\right] & =f_{i}\end{cases}
$$

By Definition of the Canonical Factors, $f_{i+1}$ does not occur in $s$ before position $x_{i+1}\left(x_{i+1}>y_{i}\right)$. We have $w_{i}=v^{\prime} f_{i+1} u$ and $w_{i+1}=v^{\prime} f_{i+1} u^{\prime}$ with $v^{\prime}=s\left[1 . . x_{1}-\right.$ $1] s\left[y_{1} \ldots x_{2}-1\right] \ldots s\left[y_{i} . . x_{i+1}-1\right]$ and $f_{i+1} u=u^{\prime \prime} f_{i+1} u^{\prime}\left(u^{\prime \prime} \in \Sigma^{+}\right)$.

Considering the contraction $\left(x_{i+1}, y_{i+1}\right)$, we have $|s|-\left|f_{i+1} u\right|+1=x_{i+1}$ and $|s|-\left|f_{i+1} u^{\prime}\right|+1=y_{i+1}$ (because the contractions are in ascending order). The word $s$ is then not yet modified after positions $x_{i+1}$, so $f_{i+1} u$ and $f_{i+1} u^{\prime}$ are suffixes of $s$. Consider the state $q=\operatorname{State}\left(f_{i+1}\right)$ of $S O(s)$, according to Lemma 7 :

$$
\operatorname{State}\left(v^{\prime} f_{i+1}\right)=q \text {. }
$$

The suffix $f_{i+1} u^{\prime}$ of $s$ is necessarily recognized by $S O(s)$. So the path accepting this suffix in $S O(s)$ go through the state $q$. Starting from $q$, we can read $u^{\prime}$ and 
reach a final state and therefore, according to Eq. (6), $S O(s)$ also accepts the word $w_{i+1}=v^{\prime} f_{i+1} u^{\prime}$. Finally, the property $(P)$ is true for all $i(0 \leq i<|\mathcal{C}|)$ and since $w_{0}=s$, we can prove by induction on $i$ that the Suffix Oracle of $s$ recognizes all words $w_{i}(0 \leq i \leq|\mathcal{C}|)$. Lemma 6 allows to conclude that each suffix of words from $\mathcal{E}(s)$ is recognized by $S O(s)$.

' $\Leftarrow$ ': Each word recognized by the Suffix Oracle of $s$ is suffix of a word from $\mathcal{E}(s)$.

Let $w$ be a word accepted by the Suffix Oracle of $s$ and $t$ be the longest suffix of $s=t^{\prime} t\left(t, t^{\prime} \in \Sigma^{*}\right)$ beginning with $w[1]$. Then a set $\mathcal{C}$ of contractions such that $t^{\prime} w=W o r d\left(t^{\prime} t, \mathcal{C}\right)$ exists (Lemma 9). Since the word $w$ is suffix of $t^{\prime} w$ and $t^{\prime} w \in \mathcal{E}(s)$, each word accepted by $S O(s)$ is a suffix of a word from $\mathcal{E}(s)$.

On the basis of Theorem 1 we give a similar result, which is available for Factor Oracles instead of Suffix Oracles.

Theorem 2 Exactly all factors of words from $\mathcal{E}(s)$ are recognized by the Factor Oracle of $s$.

Proof.

' $\Rightarrow$ ': Each factor of words from $\mathcal{E}(s)$ is recognized by the Factor Oracle of $s$.

Let $S O(s)$ be the Suffix Oracle of $s, u \in \mathcal{E}(s)$ and $m$ a factor (i.e. a prefix of a suffix) of $u=m v\left(v \in \Sigma^{*}\right)$. Then $m v$ is accepted by $S O(s)$ (Theorem 1). Thus, a path $\left(e_{0} \rightarrow e_{x_{1}} \rightarrow \ldots \rightarrow e_{x_{|m v|}}\right)$ exists in $S O(s)$, which recognizes $m v$. Therefore, we conclude that $m$ labeled a path $\left(e_{0} \rightarrow e_{x_{1}} \rightarrow \ldots \rightarrow e_{x_{|m|}}\right)$ (with $e_{x_{|m|}}$ final).

' $\Leftarrow$ ': Each word recognized by the Factor Oracle of $s$ is factor of a word from $\mathcal{E}(s)$. Let $S O(s)$ be the Suffix Oracle of $s$ and $m$ a word accepted by $F O(s)$. If $S O(s)$ recognizes $m$ then $m$ is a suffix of a word from $\mathcal{E}(s)$ (Theorem 1). Suppose that $S O(s)$ does not recognize $m$. Then $F O(s)$ recognizes $m$ at state $e_{x_{|m|}}$ (not final in $S O(s))$. Furthermore, the path $\left(e_{0} \rightarrow e_{1} \rightarrow \ldots \rightarrow e_{|s|}\right)$ exists in $O(s)$ and $e_{|s|}$ is final in $S O(s)$. We conclude that a path from $e_{x|m|}$ to $e_{|s|}$ exists in $S O(s)$. Then, the word $m$ is prefix of a word recognized by $S O(s)$ and therefore $m$ is prefix of a suffix of some $u \in \mathcal{E}(s)$. Thus, $m$ is a factor of a word of $\mathcal{E}(s)$.

\section{Properties upon Oracles \& Future Works}

According to Cleophas \& al. [11], the Oracle is not minimal in number of transitions among the set of homogeneous automata. Furthermore, if we consider the set of homogeneous automata, which recognize at least all factors (resp. suffixes) of $s$ and which have the same number of states and at most the same number of transitions than the Factor (resp. Suffix) Oracle, we show that the Oracle is not minimal on the number of accepted words. The Oracle of axttyabcdeatzattwu (see figure 6) has 35 transitions, the Factor Oracle accepts 247 words and the Suffix Oracle accepts 39 words. Though another homogeneous automaton (see figure 7), which recognizes at least all factors (resp. suffixes) of axttyabcdeatzattwu and which has only 34 transitions exists. The "Factor" version of this automaton recognizes only 236 words and its "Suffix" version accepts only 30 words. Moreover, we provide an example which has both less transitions, and less accepted words than the corresponding Oracle. 


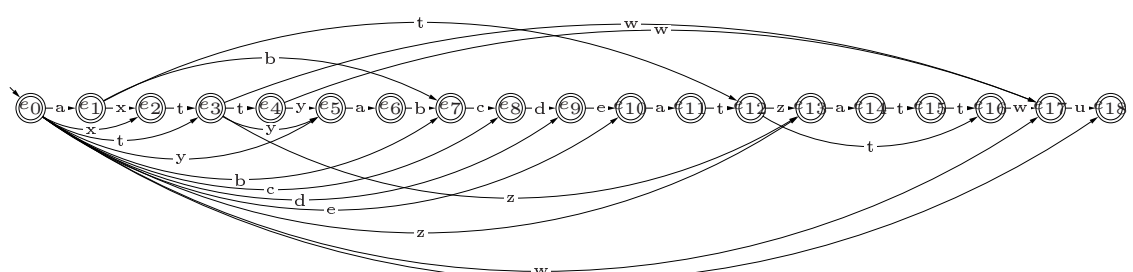

Fig. 6. Factor Oracle of the word axttyabcdeatzattwu.

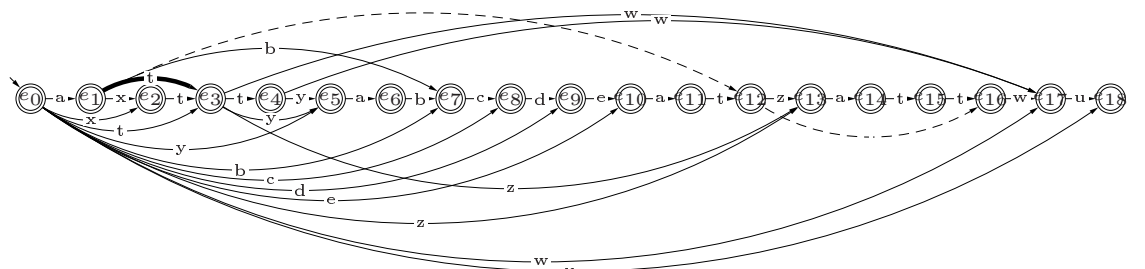

Fig. 7. This automaton (considering only the continuous lines) accepts all factors of the word axttyabcdeatzattwu. The bold transition (from $e_{1}$ to $e_{3}$ ) is the only one, which is not present in the Factor Oracle of this word (see figure 6) though the two dotted ones (from $e_{1}$ to $e_{12}$ and from $e_{12}$ to $e_{16}$ ) are present in the Factor Oracle, but not in this automaton.

In some cases, we observe that the number of words accepted by Oracles does not allow confidence to this structure when it is used to detect factors or suffixes of words. Even if the number of false positive can sometimes be equal to 0 (e.g. aaaaaa ...), it can also be exponential. Indeed, we can build a word $s$ such that each subset of $\mathcal{C}_{s}^{*}$ is coherent and minimal. For example: $s=$ aabbccddee..., the set $\mathcal{C}_{s}^{*}$ of contractions, which are available on such a word, is $\{(1,2),(3,4), \ldots,(|s|-$ $1,|s|)\}$. If we consider any non-empty subset $\mathcal{C} \subseteq\left(\mathcal{C}_{s}^{*} \backslash\{(1,2)\}\right)$ of contractions, it is easy to notice that $W \operatorname{ord}(s, \mathcal{C}) \notin F a c t(s)$. Besides, all words obtained from such subsets are pairwise different.

Number of subsets is: $\sum_{i=1}^{\left|\mathcal{C}_{s}^{*}\right|-1}\left(\begin{array}{c}\left|\mathcal{C}_{s}^{*}\right|-1 \\ i\end{array}\right)=\sum_{i=1}^{\frac{|s|}{2}-1}\left(\begin{array}{c}\frac{|s|}{2}-1 \\ i\end{array}\right)=2^{\frac{|s|}{2}-1}-1$. Then, the number of words, which are accepted by the Oracles and are not factor/suffix of $s$, is $\mathcal{O}\left(2^{|s|}\right)$.

To better use this structure, we need to improve or to slightly modify it. However, better knowledge about the Oracle structure would be useful for future works. Indeed, it could be interesting to have an empirical or a statistical estimation of the accuracy of the Oracle (time and quality of the results), when it is substituted to Tries or Suffix Trees in algorithms.

\section{Acknowledgments}

We would like to thank Céline BARRÉ to have improved the English writing of this article. We also thank Irena Rusu for its useful comments and to have improved earlier versions of this article. 


\section{References}

1. D. Gusfield, Algorithms on Strings, Trees, and Sequences: computer science and computational biology (Cambridge University Press, 1997).

2. A. Blumer, J. Blumer, D. Haussler, A. Ehrenfeucht, M. T. Chen and J. Seiferas, "The smallest automaton recognizing the subwords of a text," Theoret. Comput. Sci. 40 (1985) 31-55.

3. E. Ukkonen, "On-line construction of suffix trees," Algorithmica 14 (1995) 249-260.

4. C. Allauzen, M. Crochemore and M. Raffinot, "Factor oracle, Suffix oracle," Technical Report 99-08, Institut Gaspard-Monge, Université de Marne-la-Vallée, 1999.

5. C. Allauzen, M. Crochemore and M. Raffinot, "Factor Oracle: A New Structure for Pattern Matching," Conference on Current Trends in Theory and Practice of Informatics, 1999, pp. 295-310.

6. C. Allauzen, M. Crochemore and M. Raffinot, "Efficient Experimental String Matching by Weak Factor Recognition," Proc. of the $12^{\text {th }}$ conference on Combinatorial Pattern Matching, Lecture Notes in Comput. Sci. 2089 (2001) 51-72.

7. A. Lefebvre and T. Lecroq, "Computing repeated factors with a factor oracle," Proc. of the $11^{\text {th }}$ Australasian Workshop On Combinatorial Algorithms, eds. L. Brankovic and J. Ryan (Hunter Valley, Australia, 2000) pp. 145-158.

8. A. Lefebvre and T. Lecroq, "A heuristic for computing repeats with a factor oracle: application to biological sequences," International Journal of Computer Mathematics 79 (2002) 1303-1315.

9. A. Lefebvre, T. Lecroq, H. Dauchel and J. Alexandre, "FORRepeats: detects repeats on entire chromosomes and between genomes," Bioinformatics 19 (2003) 319-326.

10. A. Lefebvre and T. Lecroq, "Compror: on-line lossless data compression with a factor oracle," Information Processing Letters 83 (2002) 1-6.

11. L. Cleophas, G. Zwaan and B. Watson, "Constructing Factor Oracles," Proc. of the $3^{\text {rd }}$ Prague Stringology Conference, 2003. 